\title{
Beiträge zur Kenntnis der Kramenzelkalke und ihrer Entstehung.
}

\author{
Von 0. H. Schindewolf (Marburg).
}

Mit Tafel I und II.

\begin{abstract}
"Bei der Erklärung naturwissenschaftlicher Probleme wird gar zu leicht eine für richtig anerkannte Deutung einseitig verallgemeinert, während doch in der Tat die Natur äuBerst vielseitig in ihren Mittsln ist und dieselbe oder ähnliche Erscheinungen auf gar verschiedene Weise hervorzubringen imstande ist.o

G. BERENDT, 1)
\end{abstract}

\section{Vorwort.}

Im Verlaufe meiner stratigraphischen Untersuchungen über das Oberdevon in der Umgegend von Hof habe ich mich vielfach auch mit dem Gesteinsmaterial dieser Formation beschäftigt, das sich vorwiegend aus Kramenzelkalken zusammensetzt, und habe versucht, mir über das Wesen und die Entstehungsbedingungen dieser Gesteine ein Urteil zu bilden. Mannigfache Hypothesen sind bereits zur Erklärung des eigenartigen Gefüges der Kramenzelkalke aufgestellt worden, die jedoch, da sie $\mathrm{m}$. E. auch teilweise auf irrigen Voraussetzungen beruhen, nur wenig befriedigen und den tatsächlichen Verhältnissen nicht in ihrer Gesamtheit gerecht zu werden vermögen.

Es ist hier nun nicht meine Absicht, die Frage nach der Kramenzelkalkbildung in ihrem ganzen Umfange wieder aufzurollen und in historischer Reihenfolge alle die im Laufe der Zeit entwickelten Auffassungen über diesen Gegenstand zu behandeln; in annähernder Vollständigkeit ist dies bereits in den weiter unten noch des genaueren zu besprechenden Arbeiten Bokns geschehen. Es seien vielmehr im folgenden nur die Haupttheorien herausgegriffen und kritisch beleuchtet, wobei sich Gelegenheit ergeben wird, die Resultate eigener Untersuchungen hineinzuflechten. Dieser Teil meiner Arbeit wird viel Negatives enthalten; zum Schluß soll dann. versucht werden, durch Kombination der brauchbaren Kerne dieser verschiedenen Lehren und fußend auf den eingestreuten Mitteilungen über eigene Beobachtungen eine Auffassung zu begründen, die die Mannigfaltigkeiten der Erscheinungen eher zu erfassen geeignet sein dürfte, als die einzelnen bisherigen, meist auf lokale Verhältnisse zugeschnittenen Hypothesen.

Zweckmäßig hat hier eingangs eine Definition des Begriffes $\gg \mathbf{K r a}$ menzelkalk " und der gleichbedeutenden Termini "Knoten-« oder "Knollenkalk « Platz zu finden, jedoch ergibt sich vorderhand die

1) G. BERENDT, Über Riesentöpfe und ihre allgemeine Verbreitung in Nord. deutschland. Zeitschr. d. dtsch. geol. Ges. 1880. S. 66. 
Unmöglichkeit einer exakten Begriffsbestimmung; inwieweit eine solche überhaupt aufzustellen ist, wird sich erst im Verlaufe der nachfolgenden Betrachtungen zeigen. Vorläufig genügt hier die Angabe, daß man unter Kramenzelkalk einen Kalkstein versteht, der in \pm dichter Aufeinanderfolge von Tonflasern wechselnder Stärke durchzogen wird, die die derart in einzelne unregelmäßig gestaltete Knollen aufgelöste Kalkkomponente einhüllen und bei der Auswitterung des Kalkes als netz-. förmiges Leistenwerk erhalten bleiben. Ton und Kalk sind dabei nach Bors etwa zu gleichen Teilen an dem Aufbau des Kramenzelkalkes beteiligt. Bei einem Überwiegen der tonigen Komponente spricht man sinngemäß von "Kramenzel-« oder "Knollenschiefer «. Derartige Kramenzelkalke und -schiefer sind wohl ganz auf das Paläozoikum beschränkt und finden sich da im Silur, Devon und Karbon. Thre Hauptverbreitung fällt jedoch ins Oberdevon, und auf solche Vorkommnisse beziehen sich zumeist die nachfolgenden Zeilen.

Zur Übersicht sei weiterhin noch vorausbemerkt, daß sich zur Erklärung der Kramenzelstruktur in der Hauptsache zwei Gruppen von Hypothesen gegenüberstehen, die einerseits einer sekundären, posthumen und anderseits einer primären, syngenetischen Bildungsweise der fraglichen Gesteine das Wort reden.

\section{Verzeichnis der zitierten Literatur.}

1. AbEge, Handbuch dér anorganischen Chemie. II. 2. Leipzig 1905.

2. ANDRÉE, K., Über stetige und unterbrochene Meeressedimentation, ihre Ursachen, sowie über deren Bedeutung für die Stratigraphie. N. Jahrb. f. Min. usw. Beil.-Bd. XXV. 1908. S. 366-421.

3. Borr, A., Die geologischen Verhältnisse des Oberdevons im Aeketal (Oberharz). N. Jahrb. f. Min. usw. Beil.-Bd. XXXIV. 1912. S. 553-632.

4. - Über die Entstehung von Kramenzelkalk. Jahresber. d. Niedersächs. geolog. Vereins. V. 1913. S. 1-7.

5. CAYEUX, L., Introduction à l'Étude Pétrographique des Roches Sédimentaires. Mém. pour servir à l'explication de la carte géolog. détaillée de la France. Paris 1916.

6. Frech, Fr., Lethaea palaeozoica. 2. Bd. Stuttgart 1897-1902.

7. Grinxrz, H. B., Die Versteinerungen der Grauwackenformation in Sachsen und den angrenzenden Länderabteilungen. Teil II. Leipzig 1853.

8. G̈̈MBEL, C. W., Über Clymenien in den Übergangsgebilden des Fiohtelgebirges. Palaeontographica XI. 1863. S. 1-81.

9. - Geognostische Beschreibung des Königreichs Bayern. 3. Fichtelgeb. Gotha 1879.

10. LIEBE, K. Tr., Übersicht über den Schichtenaufbau Ostthüringens. Abhandl. Preu B. geolog. Landesanst. V, 4. 1884.

11. REIs, O. M., Über Stylolithen, Dutenmergel und Landschaftenkalk (Anthrakolith z. T.). Geognost. Jahreshefte XV. 1902. S. 166.

12. Reuther, L., Die Ausbildung des oberen braunen Jura im nördI. Teile der Fränkischen Alp. Geognost. Jahreshefte XX. 1907.

13. RichтеR, R., Beitrag zur Paläontologie des Thüringer Waldes I. Denkschr. d. math.-nat. Klasse d. kais. Akad. d. Wissensch. XI. Wien 1856. S. 87-138.

14. Rotr, J., Allgemeine und Chemische Geologie. I. Berlin 1879. 
15. RothPLetz, A., Über eigentümliche Deformationen jurassischer Ammoniten durch Drucksuturen und deren Beziehung zu den Stylolithen. Sitzungsber. d. math.-phys. Klasse d. kgl. bayr. Akad. d. Wissensch. 30. Heft I. 1900. S. 7-32.

16. TrwtzE, O., Über die devonischen Schichten ron Ebersdorf unweit Neurode in der Grafschaft Glatz. Palaeontographica XIX. I.871.

17. WAGNER, G., Stylolithen und Drucksuturen. Geolog. u. paläontolog. Abhdlgn. N. F. XI. Heft 2, I. 1913.

18. Walthen, K., Beiträge zur Geologie und Paläontologie des älteren Paläozoikums in Ostthüringen. 1. Das Mitteldevon und untere Oberdevon. N. Jahrb. f. Min. usw. Beil.-Bd. XXIV. 1907. S. 221-313.

19. WeBER, M., Über Bildung von Flaserkalken. Geognost. Jahreshefte. XXIV. 1911. S. $215-220$.

20. WEDEKIND, R., Die Goniatitenkalke des unteren Oberdevons von Martenberg bei Adorf. Sitzungsber. d. Ges. naturforsch. Freunde, Berlin 1913. Nr. 1. S. $23-77$.

21. - Über die Grundlagen und Methoden der Biostratigraphie. Berlin 1916.

22. ZIRKke, Lehrbuch der Petrographie. III. 1894.

\section{Hypothesen zur posthumen Entstehungsweise der Kramenzelkalke.}

\section{a) Die Auffassung Borns.}

Wohl am eingehendsten hat sich BorN ( 3 u. 4) in zwei Arbeiten mit der Frage nach der Entstehung der Kramenzelkalke beschäftigt. Er ist zugleich der überzeugteste Vertreter der Posthumitätshypothese. Folgen wir zunächst seinen Gedankengängen, die in folgenden Leitsätzen über die Mechanik der Kramenzelkalkbildung gipfeln (4, S. 7): »Die Kalkschichten des Oberdevons wurden bei der Aufrichtung des Variskischen Gebirges bis in das feinste zerklüftet. Auf diesen Klüften hat, nachdem die Kalkschichten für die Wässer der Niederschläge erreichbar waren, der Vorgang der Kramenzelbildung begonnen. Von den Spaltflächen aus lösten die Wässer, jedes. Minimum an Dichte und Kohäsion des Kalkes ausnutzend, den Kalk aus, und hinterließen die infolge stetiger Auslaugung dauerñ stärker werdenden Tonschichten, zwischen welchen die das charakteristische Bild verursachenden Kalkknollen übrigblieben, mehr oder weniger groß, je nachdem der Auflösungsprozeß vorgeschritten war «.

Als vexakten Beweis « dieser Auffassung sieht BorN seine Laboratoriumsversuche an, die zum Ziele hatten, aufzuklären, inwiefern die liegenden Adorfer Kalke seines Arbeitsgebietes, des Aeketales im Oberharz, weniger geeignet zur Bildung der Kramenzelstruktur waren als die hangenden Clymenienkalke. Es zeigte sich nämlich, daß die Flaserung in dem Profile vom Liegenden zum Hangenden zunimmt; und wenn man wie Bopus auf dem Standpunkte der posthumen Entstehungsweise der Kramenzelkalke steht, so drängt sich die Frage zur Beantwortung auf, weshalb das eine Material eher als das andere zur nachträglichen Herausbildung der charakteristischen Struktur neigte. Diese Ver- 
schiedenheit des Verhaltens kann naturgemäß nur in dem Materiale selbst begründet sein; denn die Schichten waren in gleicher Weise dem Gebirgsdruck und der Auflösungsmöglichkeit, diesen beiden nach BonN wirksamen Agentien, ausgesetzt. Eine chemische Untersuchung des Profils führte nun zu dem Resultat, daß nach dem Hangenden zu der Gehalt des Kalkes an in Wasser unlöslichen Bestandteilen, zur Hauptsache Ton, zunimmt. Darin wäre also der unterscheidende Faktor zu erblicken.

Um nun zu zeigen, daß wirklich der tonreichere Kalk zur Bildung der Kramenzelstruktur geeigneter, d. i. nach dem Gedankengange BoRNs in Wasser leichter bzw. schneller löslich ist, wurden von diesem Autor zwei Versuche angestellt. Im ersten Falle wurden zwei Würfel von je $1 \mathrm{ccm}$ Größe, einer aus dem Kalk der Clymenienzone und der andere aus dem Adorfer Kalk, in je $100 \mathrm{ccm}$ destillierten Wassers bei einer konstanten Temperatur von $20^{\circ} \mathrm{C}$ während einer Zeit von 5 Monaten schwebend aufgehängt und alsdann die Werte für die in Lösung gegangene Substanzmenge gemessen. Danach sollen von dem Clymenienkalkwürfel pro $190,284 \mathrm{mg} \mathrm{CaCO}_{3}$ und von dem Adorfer Kalkwürfel pro $180,757 \mathrm{mg} \mathrm{CaCO}_{3}$ gelöst worden sein, was einer Differenz von $12 \%$ entspricht.

Bei dem zweiten Versuche war die Anordnung eine ähnliche, mit dem Unterschiede jedoch, daß entsprechende Würfel in der gleichen Menge jetzt aber "dauernd mit Kohlensäure gesättigten « Wassers bei $10^{\circ} \mathrm{C} 4$ Monate läng aufgehängt wurden. Dabei ergaben sich folgende Zahlen für die gelösten Kalkmengen: Clymenienkalkwürfel pro 1 $176,8 \mathrm{mg} \mathrm{CaCO}_{3}$, Adorfer Kalkwürfel pro 1 134,67 mg $\mathrm{CaCO}_{8}$. Der Unterschied beträgt hier $24 \%$. Diese beiden Versuche würden nun tatsächlich, die Richtigkeit ihrer Ausführung sowie ihrer Grundlagen vorausgesetzt, eine 》bedeutend größere Löslichkeit bzw. Lösungsgeschwindigkeit des stark verunreinigten Clymenienkalkes anzeigen «.

Bei näherer Prüfung jedoch stellt sich heraus, daß den Experimenten BoRNs nur geringe Beweiskraft innewohnt; denn die von diesem Autor angegebenen Werte weichen so erheblich von den in der Literatur verzeichneten und auf genauen Messungen beruhenden Zahlen ab, dab sie keinerlei Vergleiche zulassen.

Nach einer Einwirkung des Lösungsmittels von 5 bzw. 4 Monaten darf angesichts der geringen zur Verwendung gekommenen Wassermenge, sowie der nur kleinen zur Sättigung reinen Wassers notwendigen Kalkquantitäten füglich angenommen werden, daß die Lösungen gesättigt waren, und dafür gelten nach den recht gut miteinander übereinstimmenden Angaben von ABEGG (1, S. 157), C CAYEUX (5, S. 211), Rотн (14, S. 48) u. a. folgende Zahlen: $13,1 \mathrm{mg} \mathrm{CaCO} \mathrm{CO}_{3}$ im Falle destillierten Wassers und $1300 \mathrm{mg}$ für Wasser, das mit Kohlensäure gesättigt ist. Das sind Werte, die nur $1 / 6$ bzw. über das 7 fache der von BoRN erhaltenen Zahlen betragen. Worin der Fehler der BoRNschen Unter- 
suchungen liegen mag, kann hier nicht genauer festgestellt werden, da nähere Angaben über die Versuchsanordnung fehlen. Vermutlich wird es sich jedoch bei den ersten Parallelversuchen darum handeln, daß das Wasser kohlensäurehaltig, im zweiten Falle aber nicht stetig mit Kohlensäure gesättigt war.

Die ganz unzuverlässigen Zahlen können daher in keinerlei Weise zv Argumentationen herangezogen werden. Ein weiterer Grund tritt hinzu: bereits oben konnte - wenigstens für den ersteren der Versuche eine Sättigung des Lösungsmittels als gesichert hingestellt werden, und auch insofern dürfen die von BoRN angegebenen Differenzen nicht als Ausdruck der verschiedenen Lösungsgeschwindigkeit des Kalkes in den beiden Kalkwürfeln angesehen werden. Daß übrigens Tonbeimengungen einem Kalke infolge einer "Verminderung der Kohäsion * und 》der großen Aufnahmefähigkeit des Tons für Wasser « eine um etwas vergrößerte Lösungsgeschwindigkeit verleihen können, sei gern eingeräumt; nur einen sexakten Beweis « für diese Tatsache und besonders in einem derart weiten Umfange vermag ich nicht mehr in den Borsschen. Versuchen zu erblicken.

Überraschend ist weiterhin an den mitgeteilten Versuchszahlen, daß die Gewichte der in 11 gelösten Kalkmengen teilweise bis auf tausendstel Milligramme angegeben werden. Da die experimentelle Bestimmung mit $100 \mathrm{ccm}$ Wasser durchgeführt wurde, so müssen die Originalmessungen noch um eine weitere Dezimale genauer_sein. Es würde großem allgemeinen Interesse begegnen, Genaueres über die angewandten analytischen Methoden wie über die praktische Durchführung solcher Wägungen zu erfahren, deren Genauigkeitsgrad meines Wissens die bisher bekannten Hilfsmittel noch nicht zu erreichen gestatteten.

Nachdem so die Hypothese BorNs ihrer bestechenden Grundlage und mithin ihrer Hauptstütze beraubt werden mußte, erscheint es am Platze, weitere Einwände vorzubringen, die sich nicht mit ihr vereinigen lassen bzw. deren Unwahrscheinlichkeiten dartun.

Eine schwache Seite seiner Lehre hat BoRN bereits selbst erkannt; sie beruht in der überraschenden Horizontbeständigkeit und weiten horizontalen Verbreitung der oberdevonischen Kramenzelkalke, die sich nicht nur in den zahlreichen alten Gebirgen Europas; sondern ebenso auch in Nordamerika finden. Angesichts dieser Tatsache spricht m. E. aber nur geringe Wahrscheinlichkeit für eine Lehre, die annimmt, daß eine derart verhältnismäßig wenig mächtige Kalkfolge horizontal weithin gleichmäßig posthum in Kramenzelkalk umgewandelt sei und zwar unter Bedingungen, die nicht eben gewöhnlich zu nennen sind.

Es sei weiterhin hinzugefügt, daß eine derartige Regelmäßigkeit nicht nur in der Horizontalen, sondern auch in der Vertikalen statt hat. So besteht das Paläozoikum Thüringens aus etwa $3000-4000 \mathrm{~m}$ mächtigen Schiefergesteinen, deren petrographische Gleichartigkeit derart ist, daß man den ganzen Schichtenkomplex als Schiefergebirge bezeichnet 
hat. Diesem Schiefersystem sind nun in drei verschiedenen Niveaus Kalke eingeschaltet: im Obersilur die Ockerkalke, an der Basis des Mitteldevons die Tentaculiten-Knollenkalke (oder nach K. WALTHER (18, S. 249) zweckmäBiger als Knollenschiefer zu bezeichnen) und im Oberdevon die Goniatiten- und Clymenienkalke, die alle gleichartig als Knollenkalke ausgebildet sind. Sollten da nun, so frage ich, überall posthume Vorgänge zur Herausbildung der Knollenstruktur geführt haben, oder hat nicht vielmehr die Auffassung größere Wahrscheinlichkeit für sich, die in diesem Falle eine primäre Entstehungsweise in Anspruch nimmt?

Ist durch das Vorhergehende einer mehr gefühlsmäßigen Stellungnahme zu der BoRNschen Hypothese Ausdruck gegeben worden, so wird durch eine weitere Tatsache schlagend vorerst die Auffassung widerlegt, daß die tonigen Mittel der Kramenzelkalke in ihrer Gesamtheit sekundärer Anreicherung und Ablagerung sind. Es ist das die Fossilführung der Tonflasern, die auch ausdrücklich von BonN (3, S. 625) erwähnt wird. Diese Erscheinungsweise spricht nun $\mathrm{m}$. E. unbedingt für eine primäre Ablagerung; wenigstens erscheint es mir unvorstellbar, wie etwa die zarten Schalen von Posidonia venusta, um die es sich dabei vornehmilich handelt, bèi der Konzentration des Tongehaltes aus dem Kalke heraus. gelöst und dann unversehrt wieder in die Schiefer eingebettet sein sollten.

Weiterhin finden sich dann durch Zunahme des Tongehaltes alle Übergänge zwischen nur'schwach geflaserten Kramenzelkalken. (Flaserkalken im Sinne Bonss) zu typischen Kramenzelkalken, dann zu. Kramenzelschiefern mit nur vereinzelten Kalkknollen und schließlich zu reinen Tonschiefern, eine Erscheinungsweise, für deren Erklärung später eine einheitliche Formel gesucht werden muß, nachdem die Beweise für die Deutung BoRNs nicht als richtig haben anerkannt werden können. Gerade diese enge Verknüpfung mit Bildungen, die zweifellos eine durchaus andere Erklärungsweise als die BonNs erheischen, läßt mich im Anschluß an R. Wedekind (20, S. 35), der vor einer Reihe von Jahren. bereits auf diese Verhältnisse hinwies und daran auch die Unwahrscheinlichkeiten der Borsschen Hypothese darlegte, weiterhin zu deren Ablehnung gelangen.

Ebenso wird sich auch später eine einfache Erklärung für die auf den ersten Blick eigenartig erscheinende Gebundenheit der stärkeren Flaserung an Kalke von größerem Tongehalt ergeben.

Im übrigen sei noch darauf hingewiesen, daß Bildungsbedingungen, wie sie streng nach dem Wortlaut BoRNs angenommen werden müssen, unvorstellbar erscheinen. Nach ihm wurden die Kalkschichten des Oberdevons "bei der Aufrichtung des Variskischen Gebirges bis in das feinste zerklüftet «, und »auf diesen Klüften hat, nachdem die Kalkschichten für die Wässer der Niederschläge erreichbar waren, der Vorgang der Kramenzelbildung begonnen «. Nun sind jedoch vielfach die Tonflasern von einer derartigen Feinheit, daß man sich nicht vorzustellen 
vermag, wie auf einem vorher an deren Stelle existierenden kapillaren Spaltensystem eine Zirkulation von Wässern stattfinden konnte. Man hätte vielmehr anzunehmen; daß die Bildung der Tonflasern unter einem ständig wirkenden Drucke vor sich ging, der seine Auswirkung hauptsächlich längs der Spalten fand und zu deren nachträglicher Verengung führte, wie man sich auch neuerdings die Stylolithen und Drucksuturen als Rückstände von Lösungsvorgängen vorstellt, die unter Kompression im festen Gestein statthatten ${ }^{1}$ ).

Die BonNsche Posthumitätslehre käme also auf nichts anderes heraus, als daß die Tonflasern - in ganz ähnlichem Sinne hat sich auch BoRN bereits selbst ausgesprochen - auf Drucksuturen innerhalb der Kalke zurückgeführt werden und diesen die Rolle des allein formgebenden Elementes bei der Kramenzelkalkstruktur eingeräumt wird. Daß dies aber zu Unrecht geschieht, konnte oben bereits auf verschiedenen Wegen nachgewiesen werden, und im folgenden werden noch weitere Einwände dagegen zu erheben sein. Nun fehlen allerdings, wie auch ich anerkenne, Drucksuturen und diesen ähnliche Gebilde den Kramenzelkalken keineswegs ${ }^{2}$ ); sie finden sich nach RöthPLETz (15, S. 13) win allen Kalksteinen aller Formationen, wo mehr oder weniger starke Aufrichtung und Faltung des Sedimentes stattgefunden hat «, und es wäre danach direkt auffällig, wenn die Kramenzelkalke, die vielfach den genannten Bedingungen entsprechen, frei davon wären.

Durch eine derartige Drucksutur ist das Bild des von Born in 3, Taf. XXI, Fig. 10 dargestellten Goniatiten entstanden zu denken. Da dergleichen Fälle aber nur äußerst selten vorkommen, kann den Drucksuturen bei weitem nicht die Bedeutung zukommen, die ihnen BoRN zuschreibt. Nach meinen Beobachtungen im fränkischen Oberdevon, sowie dem von Ostthüringen, Schlesien, des Harzes, des Rheinlandes und anderer Gegenden ragen die Fossilien, wenn sie innerhalb der Schichtfläche gerade auf der Grenze von Kalk und Tonmittel gelegen sind, in weitaus der überwiegenden Mehrzahl der Fälle unversehrt und plastisch in dieses hinein, ohne irgendwie von der Tonflaser durchquert oder sonst von ihr beeinflußt zu werden. Sehr häufig konnte aber fermerbin auch beobachtet werden, daß die Fossilien, namentlich Clymenien, nicht parallel zur Schichtebene, sondern senkrecht oder unter einem mehr oder weniger spitzen Winkel zu ihr liegen und infolge dieses Umstandes

1) G. WAGNer (17): "Stylolithen, Drucksuturen und Eindrücke bei Geröllen entstehen durch chemische Auflösung unter Druck im festen Gestein «. Dabei geben die Stylolithenzüge stets die Richting senkrecht zum Druck an.

2) Merkwürdig allerdings muß es bleiben, daß in den Kramenzelkalken meines Wissens niemals bisher Stylolithen beobachtet worden sind, die in ihrem Auftreten und ihren Entstehungsbedingungen auf das engste mit den Drucksuturen verknüpft zu sein pflegen und nach WAGNor (a. a. O.) eigentlich nur eine graduell verschiedene Ausbildungsform von jenen darstellen. Nach demselben Autor findet man bei sonstigen Kalken shäufig im selben Handstück Drucksuturen und Stylolithen mit allen möglichen Übergängen s. 
mehrere Tonflasern kreuzen. Stets war dann das Verhältnis derart, daß die Tonflasern deutlich an den betreffenden Fossilien absetzen bzw. sich um diese herumlegen, niemals äber durch sie hindurchgehen. Wenn aber "zahlreiche Fossilien der Schale entbehren und statt ihrer von einer schwärzlichen Tonschicht umgeben sind « $(3$, S. 629), so erklärt sich dieser Sachverhalt m. E. zwanglos durch die Korrosion des Meerwassers ${ }^{1}$ ), dazu ist nicht die Annahme sekundärer Einwirkung als Kluftbildung usw. notwendig.

Eine weitere Beobachtung erscheint mir alsdann in diesem Zusammenhange der Beachtung wert. Nach BorN ist als auslösende Kraft bei der Bildung der Kramenzelkalke die Aufrichtung des Variskischen Gebirges tätig. Nun zeigen häufig die Kramenzelkalke wie namentlich die Knotenschiefer eine transversale Schieferung, was besonders typisch u. a. am Bohlen bei Saalfeld, bei Bicken in der Dillmulde und bei Grevenbrücck in der Attendorn-Elsper Doppelmulde von mir beobachtet werden konnte. Diese tektonische Beeinflussung, die doch zweifellos durch die variskische Faltung hervorgebracht ist und uns in so hervorragender Weise die ehemals obwaltenden Druckverhältnisse widerspiegelt, steht nun aber in keinerlei Beziehung zu der Flaserung der Kalke, indem die Anordnung der Kalkknollen und der Tonflasern eine streng parallele im Sinne der Schichtung ist, die dann unter einem \pm großen Winkel von der Schieferung durchschnitten wird. Wäre die Bildung von Drucksuturen das ausschlaggebende Formelement bei der Herausprägung der Kramenzelkalkstruktur, so müßte man jedoch erwarten, daß die Tonflasern senkrecht zum Druck, also in der Schieferungsebene, angeordnet wären. Da dies nicht der Fall ist, gelangen wir auch auf diesem Wege zu. einer Ablehnung der Erklärungsweise mit Hilfe von Drucksuturen, und die Auffassung gewinnt weiterhin an Wahrscheinlichkeit, daß der Kramenzelkalk zur Zeit der variskischen Faltung bereits als solcher fertig vorgelegen hat und somit primärer Entstehung ist.

Daß es weiterhin auch Kramenzelkalke gibt, die keinerlei tektonischen Einflüssen unterlegen waren und mithin diesen auch nicht ihre Entstehung verdanken, kann ich an einem treffenden Beispiel belegen, zu dem mir meine vor einigen Jahren im Untersilur Esthlands angestellten Beobachtungen das Material an die Hand geben. Es gelang mir nämlich, innerhalb des Echinosphaeritenkalkes der Umgegend von Reval neben häufig gleichfalls netzartig verwitternden dolomitischen Kalken echte Kramenzelkalke mit allen Charakteren der oberdevonischen Bildungen (vgl. Taf. I, Fig. 1) nachzuweisen, die mir nun in ihrer absolut schwebenden Lagerung und vollkommenen tektonischen Unberührtheit einen schlagenden Gegenbeweis gegen die Theorie BorNs oder wenigstens deren allgemeine Anwendbarkeit zu liefern scheinen, die ja zu ihrer Erklärungsweise eine tektonische Mitwirkung beansprucht.

1) Vgl. dazu Fr. Frecr, 6, S. 134 und K. ANDréE, 2, S. 412. 


\section{b) Die Auffassung Webers.}

(Vgl. Taf. I, Fig. 2; Taf. II, Fig. 2.)

Eine zweite Hypothese, die gleichfalls eine posthume Erklärungsweise der Kramenzelkalke annimmt, ist von M. WEBER (19) entwickelt worden. Sie bezieht sich auf das oberdevonische Kramenzelkalklager von Horwagen im Frankenwalde, dessen Bildungsbedingungen sich WeBER (a. a. O., S. 216) folgendermaßen denkt: »Bänke von dichtem Kalke sind unter dem Einflusse von gebirgsbildenden Kräften zunächst in langgestreckte, im Querschnitt scharfeckige Schollen zertrümmert worden; diese wurden dann weiter aneinander verschoben, rundeten sich gegenseitig ab oder erhielten längliche Formen, je nachdem die Richtung des Druckes wechselte oder konstant blieb. Die tonigen Lagen dienten als Gleitflächen und als Schmiermittel, das neben dem infolge der Reibung entstehenden Abfall auch überall hineingepreßt wurde, wo sich durch Bildung von toten Winkeln Platz ergab, daher der wabenartige Bau «. Als Ausgangsmaterial der Kramenzelkalke werden von WEBER »dünnbankige Kalke mit schwächeren tonigen Zwischenmitteln * angenommen.

Seine besondere tektonische Position erhält der Horwagener Kramenzelkalk durch die unmittelbare Nachbarschaft eines mächtigen Diabas- (Spilit-) Lagers, dessen Wirkung als starres Widerlager bei dem Gebirgsdruck die Bildung der eckigen bzw. mehr gerundeten Kalkbreccien veranlaßte and eine dynamometamorphe Umwandlung des Kalkes selbst hervorrief. Die intensivste Veränderung der Kalke sowohl hinsichtlich der Abrundung der Breccie wie auch der Umkristallisation findet sich an der Berührungsstelle mit dem Diabase, wo die Auswirkung des Druckes naturgemäß am stärksten sein mußte.

Während wiederholter Besuche der Horwagener Aufschlüsse konnte ich nun die Darstellung WtBERs von einer teilweise brecciösen Natur des dortigen Kalklagers vollauf bestätigen, mußte aber weiterhin feststellen, daß der Vorgang der Breccienbildung nicht zugleich Faktor der Kramenzelkalkentstehung sein kann. Es zeigte sich vielmehr, daß die einzelnen Komponenten der Breccie bereits aus typischen Flaserkalken bestehen, die in ihrem Charakter durchaus denen der Umgegend von Hof entsprechen. Die Tonflasern sind zur Hauptsache parallel angeordnet und gehen zuweilen in drucksuturenartige Gebilde über. Zwischen je zwei parallelen Tonhäuten breitet sich fernerhin ein unregelmäßiges Netzwerk verbindender Tonflasern aus. Nach den im frischen Bruch oder Anschliff sich ergebenden Bildern wäre man vielleicht geneigt, derart vorwiegend parallel struierte und plattenkalkähnliche Gesteine nicht für echte Kramenzelkalke zu halten, jedoch tritt bei diesen, wit ich vielfach beobachten konnte, nach stattgehabter Anwitterung deutlich die Kramenzelstruktur in die Erscheinung (vgl. Taf. II, Fig. 1).

Wenn nun auch, wie wir sahen, bei kritischer Prüfung die Hypothese 
WEBERs zur Erklärung des Horwagener Kramenzelkailkes versaǵt, so ist doch immerhin die Möglichkeit ins Auge zu fassen, daß ähnliche Bedingungen anderenorts vielleicht einmal zur Herausbildung von Kramenzelkalken geführt bzw. einen gewissen Anteil daran genommen haben könnten, obwohl bisher derartige Vorkommnisse noch nicht bekannt geworden sind. In allen solchen Fällen hat man sich aber unbedingt vor einer unangebrachten Verallgemeinerung zu verwahren, und, wie das vorliegende Beispiel deutlich zeigt, ist es ein großer Fehler, wenn WEBER glaubt, mit seiner nicht als stichhaltig anzuerkennenden Lehre wahrscheinlich alle obersilurischen und devonischen Kalke des mittleren Teiles des Variskischen Bogens « erfassen zu können.

Wie oben bereits $u$. a. an dem Beispiele der untersilurischen Kramenzelkalke Esthlands gezeigt wurde, müssen außer den Bildungsbedingungen, die uns BORN und WEBER gelehrt haben, und die auch bis zu einem gewissen Grade als richtig oder wenigstens möglich anerkannt werden konnten, noch andere in der Natur vorkommen, und im folgenden werden noch weitere Möglichkeiten dafür zu entwickeln sein. Durch diese Erkenntnis bin ich zu der Auffassung einer heterogenetischen Bildungsweise der Kramenzelkalke gelangt. Wir haben daher nicht nach einer Erklärungsweise des Kramenzelkalkes zu suchen, sondern alle die theoretisch denkbaren Fälle ins Auge zu fassen und danach abzuwägen, welcher Anteil diesen einzelnen Möglichkeiten bei dem Aufbau der Kramenzelkalke zukommt, und wieweit sie überhaupt in der Natur wirklichen Ausdruck gefunden haben.

\section{Hypothesen zur syngenetischen Entstehungsweise der Kramenzelkalke.}

\section{a) Die Auffassung Richters.}

Diesen Hypothesen eines posthumen Entstehungsmodus der Kramenzelkalke stehen nun zur Hauptsache zwei weitere einer primären syngenetischen Bildungsart gegenüber, deren zuerst zu besprechende von R. Richter (13, S. 92) aufgestellt worden ist. Sie basiert lediglich auf Beobachtungen über die Saalfelder Kramenzelkalke, die als ein Kalkkonglomerat mit tonigem Bindemittel angesprochen werden. Geführt wurde RICHTER zu dieser Anschauung durch eine angeblich beobachtete Abrollung der Fossilien in den Kalkknollen und eine vermeintliche Verschiedenheit der Fauna des Schiefers von der der ihm eingelagerten Kalke, Beobachtungen, die allerdings, wenn sie sich bewahrheiteten, den Folgerungen des Autors Recht geben würden

Als Ursprungsgebiet der bei Saalfeld abgelagerten Kalkknollen sieht Richter die Oberdevonvorkommen des Frankenwaldes und des Fichtelgebirges an, die die gleichen Fossilien einschließen wie die Kalkknollen der Kramenzelkalke in der Umgegend von Saalfeld. Während Zeiten 
der Trockenlegung soll das Material dort aufbereitet worden sein, um dann bei einer Meeresüberflutung fortgeschwemmt und im Saalfelder Gebiete niedergelegt zu werden. Mehrfacher Wechsel dieser Vorgänge soll alsdann unter Zufuhr tonigen Materials als Matrix des Konglomerates die Kramenzelkalke von Saalfeld geschaffen haben.

Eingehende Untersuchungen am Bohlen und den übrigen Oberdevonaufschlüssen in der Umgegend von Saalfeld haben mir nun aber gezeigt, daß keines der oben aufgeführten Argumente Richters aufrecht zu érhalten ist. Der schlagendste Beweis gegen dessen Hypothese ist aber wohl der Umstand, daß ich innerhalb des Saalfelder: Oberdevons genau die gleiche Schichtenfolge wie im oberfränkischen Oberdevon nachweisen konnte, während man unter Bildungsbedingungen, wie sie Richter vorschwebten, doch eine Umkehrung des Profils oder wenigstens eine Vermengung von Komponenten verschiedener stratigraphischer Einheiten erwarten müßte. Auf einige weitere Unmöglichkeiten, die die Lehre Richters einschließt, hat bereits TIETze (16, S. 123) bei deren Kritik hingewiesen, Einwände, die als durchaus stichhaltig anzuerkennen sind.

Zu ganz ähnlichen Anschauungen bezüglich der Bildungsbedingungen der Kramenzelkalke neigen Gümber (8, S. 5) ${ }^{1}$ ), Geinitz (7, S. 13) und Tretze (a. a. 0.), die teils in der Kramenzelkalkstruktur den Charakter eines Brandungskonglomerates, teils den Ausdruck bewegten Wassers sehen, das die hier jedoch mehr oder weniger autochthonen Kalk- und Schieferschichten vermengte.

Welchen Grad der Wahrscheinlichkeit oder der theoretischen Möglichkeit schließen nun diese Hypothesen ein, wenn man von den bereits als unrichtig erkannten weitergehenden Folgerungen RichTERs abstrahiert? BoRN glaubte bei einer kritischen Besprechung dieser Lehren sie in ihrem vollen Umfange ablehnen zu sollen; denn »die Auffassung des Kramenzelkalkes als Produkt bewegten Wassers, wie es die syngenetische Auffassung fordert, sei es als Strandbildung, als Transgressionskonglomerat, sei es als Vermengung. von Ton- und Kalkschichten infolge sanften Wellenschlages, hat zur notwendigen Folge, daß man die Knotenkalke des Oberdevons als Ablagerung von großer Küstennähe oder eines sehr flachen Meeres anzusehen hat «. Dieses für BoRN an-

1) Es scheint allerdings, als ob GüMBEL niemals zu einer kiaren Auffassung über die Natur der Kramenzelkalke gelangt ist. Während er a. a. O. "mit Entschiedenheit « die Ansicht vertritt, daB die Kalkknollen der Kramenzelkalke an stark brandenden Meeresstellen entstandene Strandbildungen sind, lehnt er später (9, S. 296) eine konglomeratische Bildungsweise wiederum direkt ab; denn 》die Beobachtungen zahlreicher Vorkommnisse im Fichtelgebirge sprechen für ihre Entstehung nach Art der Geoden \&, oder aber ex zieht wieder an andrer Stelle (9, S. 509) einen gänzlich verfehlten Vergleich der bisweilen eisen- und manganhaltigen Kalkknollen mit den bekannten Mangan- und Eisenoxydknollen des roten Tiefseetones. 
scheinend einzig in Frage kommende Postulat wird jedoch durch die faziellen und faunistischen Eigentümlichkeiten des Oberdevons widerlegt, auf Grund deren er zu der Auffassung gelangt, daß das Oberdevonmeer mindestens eine Tiefe von $200 \mathrm{~m}$ besessen hat, eine Tiefe, bis zu welcher nach den modernsten Forschungen Wasserbewegungen der Oberfläche kaum sich fortpflanzen können «.

Müssen es nun, so wende ich ein, Wasserbewegungen der Oberfläche sein, die als die oben geforderten Kräfte in Betracht kommen, können es nicht vielmehr auch ebensogut submarine Bewegungen, tiefer liegende Meeresströmungen sein, die in der bezeichneten Weise einwirkten? Solche tieferen Meeresströmungen ${ }^{1}$ ) können dann ungefähr synchron, d. h. sofort nach Ablagerung der Schichten und vor ihrer diagenetischen. Umwandlung, die Kalk- und Tonschichten erfaßt und zu ihrer Vermengung geführt haben, oder aber, es kann auch posthum nach stattgehabter Verfestigung der Schichten eine Aufarbeitung erfolgt sein, wobei ich als natürliches Beispiel die von L. ReuTHer (12, S. 80) beschriebenen Geröllschichten des fränkischen Kellaway vor Augen habe, die man als Produkt einer submarinen Abrasion bezeichnen könnte.

Theoretisch möglich wäre also eine derartige Entstehungsart durchaus, und, wenn man mit mir auf dem Boden einer heterogenetischen Bildungsweise der Kramenzelkalke steht, darf man vielleicht annehmen, daß sie an dem einen oder anderen Orte auch tatsächlich verwirklicht sein mag. Irgendwelche Beobachtungen, die dieser Vermutung etwas mehr Gewicht verleihen könnten, sind bisher noch nicht gemacht worden. Eine intensivere Abrollung der Fossilien und der Charakter eines typischen Trümmergesteins, wie es namentlich in dem zweiten Falle der posthumen Entstehungsweise in diesem Sinne zu fordern wäre, hat bisher noch an keinem der mir bekannten Oberdevonvorkommen festgestellt werden können.

Auf der anderen Seite liegt aber, wie R. WEDEKIND (21, S. 45) zeigte, der Bildungsbereich der oberdevonischen Sedimente in weit geringeren Meerestiefen, als sie BoRN annehmen zu müssen glaubte, in Tiefen von nur etwa $50 \mathrm{~m}$, die sogar noch eine wirksame Fortpflanzung von oberflächlichen Meeresbewegungen bis auf den Meeresgrund gewährleisten würden.

Ein Erklärungsversuch der Kramenzelkalke als Transgressionskonglomerat etwa im Sinne GüMBeLs u. a. dürfte dagegen durchaus unhaltbar sein.

1) Die Existenz derartiger auch bis in größere Tiefen hinabreichender Meeresströmungen steht nach den Ergebnissen der modemen Tiefseeforschung unzweifelhaft fest. Auf Grund einer Besprechung zahlreicher diesbezüglicher Literaturangaben ergeben sich nach K. ANDRíe (2, S. 40I) "selbst für Tiefen, die $1000 \mathrm{~m}$ übersteigen, Geschwindigkeiten, welche eine mechanische Wirksamkeit ohne Zweifel auszuüben imstande sind ${ }_{*}$. 


\section{b) Die Auffassung Zirkels und deren Erweiterung zu der Arbeits- hypothese des Verfassers.}

Zum Schluß ist dann endlich noch ein von Zirknu (22, S. 469) genauer präzisiertes Theorem einer syngenetisch-chemischen Bildungsweise abzuhandeln, dem ich mich am meisten, allerdings mit mancherlei Einschränkungen und Modifikationen anzuschließen vermag; Nach ZIRKELs Gedankengange spielt bei der Entstehung der Kramenzelkalke die Tierwelt des Meeres eine große Rolle. Die Weichteile der abgestorbenen und zu Boden gesunkenen Tiere bilden bei der Verwesung Ammonium- und Natriumkarbonate, und diese gehen durch Umsetzung mit dem Kalziumsulfatgehalt des Meerwassers in kohlensauren Kalk über, der sich rings um die Tierschalen konzentrierte und so zu der Bildung der Kalkknollen Anlaß gab.

Unter Benutzung dieser Theorie sind von K. WaLther (18, S. 249) die Knollenschiefer im unteren Mitteldevon Ostthüringens erklärt worden. Auch von K. TH. IrEBE (10, S. 21), wurde bereits weit früher, wenn auch in unbestimmterer Form, die Auffassung vertreten, daß die Knotenkalke des Oberdevons ihr eigentümliches Gepräge durch das Auftreten von Kalkkonkretionen erhalten haben.

Gegen die Erklärungsweise ZrRKeLs ist nun eingeworfen worden, daß bei weitem nicht alle Kalkknollen, wịe zu fordern wäre, Fossilreste enthalten, doch halte ich diesen Finwand nicht eben für sehr treffend, wenn man sich vergegenwärtigt, daß erfahrungsgemäß doch nur ein kleiner Prozentsatz der fossilen Lebewelt günstige Erhaltungsbedingungen gefunden hat, und daß daher die vielleicht ehemals vorhandenen Fossilien in einer großen Anzahl der Fälle der Zerstörung anheimgefallen und für uns nicht mehr nachweisbar sein können.

Im übrigen lasse ich es durchaus dahingestellt, ob die von ZIRKEL namhaft gemachten Gründe für die Kalkkonzentration die einzig möglichen sind, oder ob nicht vielmehr auch noch andere Umstände zu einer konkretionären Zusammenballung des Kalkgehaltes führen können. Dagegen halte ich aber durchaus an der konkretionsartigen Natur der Kalkknollen fest, und dafür scheinen mir die folgenden Erwägungen zu sprechen.

Bereits oben wurde darauf hingewiesen, daß häufig in ein und demselben Profil alle Übergänge zwischen reinen Plattenkalken, einfach tongebänderten Kalken mit geringem Tongehalt, dann solchen mit mehr hervortretendem Tonmaterial und der typischen Kramenzelstruktur, alsdann bei weiterem Anwachsen des Tongehaltes Kramenzelschiefern und endlich reinen Tonschiefern zu beobachten sind. Ess erscheint selbstverständlich, daß angesichts der kontinuierlichen $\mathrm{Zu}$ sammenhänge, die sowohl in der Vertikalen wie auch gelegentlich in der Horizontalen auftreten, eine einbeitliche Erklärungsformel für die verschiedenen Erscheinungsarten gefunden werden muß. 
Da ist es nun in erster Linie der Habitus der in den Kramenzelschiefern vorhandenen Kalkknollen, der mir einen Vergleich mit konkretionsartigen Bildungen nahelegt. Sie besitzen in ihrer ganzen Erscheinungsweise eine derartige Übereinstimmung etwa mit den Schwefelkies- und Kalkkonkretionen, wie sie in den unterkretazischen Tonen Norddeutschlands auftreten, daß ich an ihrer analogen Entstehungsweise keine $\mathrm{Zweifel} \mathrm{mehr} \mathrm{hegen} \mathrm{kann.}$

Danach stelle ich mir die Entstehungsbedingungen eines wie oben geschilderten Profiles etwa in der folgenden Weise vor: In einem Meere, in dem jegliche Tonzufuhr fehlte, setzten sich zunächst reine Plattenkalke ab. Bei periodischer Tonzufuhr in geringem Maße bildete sich dann eine Wechsellagerung von vorwiegenden Kalken mit dünnen Tonzwischenmitteln heraus, die im kleinen die Verhältnisse darbieten, wie etwa der Tonplattenhorizont des deutschen Muschelkalkes. Die Erscheinungsweise dieser Sedimente ist dann überall eine derartige, daß kein geologisch geschulter Mensch ohne Voreingenommenheit darauf verfallen würde, ihnen eine andere als primäre Entstehungsweise zuzuschreiben, und, was für diese gilt, muß auf Grund der oben geforderten Einheitlichkeit der Erklärungsweise auch für die nun zu besprechenden Bildungen Gültiglzeit besitzen.

Bei verstärkter Tonzufuhr kontinentalen Ursprungs trat alsdann ein mehr oder weniger vollkommener konkretionärer Zusammenschluß der Kalkkomponente ein, so daß daraus primär die Erscheinungsweise \pm großer ellipsoidischer oder auch mehr plattenförmiger Kalkknollen resultierte, die in eine schiefrige Matrix eingebettet sind. Bei überwiegendem Tonmaterial gehen daraus Kalkknotenschiefer und schließlich beim Aufhören jedes kalkigen Niederschlages reine Tonschiefer hervor.

Nun erscheint es selbstverständlich, wenn eine immer mehr gesteigerte periodische Zufuhr tonigen Materials, das die Tonflasern bildete, angenommen werden muß, daß dann damit parallellaufend auch die Kalkeinlagerungen einen mehr tonigen Charakter aufweisen, da die Tonlieferung natürlich zur Zeit der Kalkausscheidung nicht ganz ausgeschaltet, sondern nur eingeschränkt war. So gelangen wir also zu der Auffassung, daß der höhere Tongehalt innerhalb der Kalke und die verstärkte Flaserung eine durchaus primäre und einheitliche Ursache besitzen, und daB nicht, wie Bons wollte, die erstere Tatsache die Vorbedingung für die zweite ist.

Die Zunahme der tonigen Komponente in den oberdevonischen Bildungen nach dem Hangenden zu, die an all den von mir untersuchten Vorkommen festgestellt werden konnte, ist nun aber der Ausdruck einer steigenden Heraushebung und Abtragung der das Oberdevonmeer beherrschenden Kontinentalmassen: wir nähern uns mit dem Ausgang des Oberdevons der variskischen Faltungsperiode, deren Vorphase in das jüngere Oberdevon fällt. Solche Landmassen, deren Heraushebung 
auch durch andere Anzeichen, wie z. B. Transgressionserscheinungen bewiesen wird, liegen nach $\mathrm{R}$. WEDEKINDs und meinen Untersuchungen namentlich in dem gewaltigen "Nordatlantischen Kontinente", der großen im Herzen von Mitteleuropa gelegenen "Alemannischen Insel « und der die podolische Platte umfassenden "Podolischen Insel « vor. Die Annahme dieser kontinentalen Gebiete ermöglicht uns von einheitlichem Gesichtspunkte aus die Erfassung der paläogeographischen Eigenheiten des Oberdevonmeeres und gibt uns auch eine Erklärung der Vorbedingungen für die Kramenzellalkbildung an die Hand.

$\mathrm{Zu}$ dieser primären Art der Herausbildung der Kramenzèlstruktur tritt dann als weiteres formgebendes Element noch die Drucksuturenbildung, der oben bereits ein geringer Anteil an dem Aufbau der Kramenzelkalke eingeräumt wurde. Die Häufigkeit des Auftretens von Drucksuturen und somit das Verhältnis zwischen primärer und sekundär angereicherter Tonsubstanz scheint ein wechselndes zu sein. So spielen anscheinend z. B. in manchen Partien der Kramenzelkalke vom Eichelberge bei Hof Drucksuturen eine größere Rolle als in den gleichaltrigen Knotenkalken von Gattendorf bei Hof. Sie können jedoch bei dem Mangel jeglicher tektonischer Einwirkung auch gänzlich fehlen, wie an dem Beispiele der wohlentwickelten Kramenzelkalke im Untersilur Esthlands gezeigt werden konnte, und sind demgemäß nur von geringem Einflusse auf die Herausbildung der Kramenzelstruktur.

Die Kramenzelkalke aber sind danach deutlich polygener Entstehung und müssen immer von Fall zu Fall geprüft werden, wenn im einzelnen etwas über ihre Entstehung ausgesagt werden soll. Gemeinsam für alle dürfte jedoch gelten, daß stets Tonsubstanz primär als solche gesondert in den Schichten vorhanden war; hinzutreten kann dann noch Druckflaserung, die eine sekundäre Tonanreicherung in dem Kalke verursachte. Einige weitere Möglichkeiten konnten fernerhin noch theoretisch abgeleitet werden, ohne daß es bisher gelungen wäre, Belege dafür in der Natur nachzuweisen.

Daraus erhellt nun, daß eine genauere allgemein gültige Definition der Kramenzelkalke über den Rahmen der oben gegebenen Richtlinien hinaus nicht möglich ist; entsprechend ihrer verschiedenen Entstehungsweise müssen sie im einzelnen innerhalb dieser Grenzen auch verschiedene Merkmale zeigen. Demgemäß hätte auch eine besondere Bezeichnungsweise der verschiedenen Kramenzelkalkarten Platz zu greifen. - Ein solcher Vorschlag zur Klassifikation ist bereits von M. WEBER (a. a. O.) gemacht worden, der den Namen »Knollenkalk « auf die primär-konkretionär entstandenen Kramenzelkalke beschränken, »die Bezeichnung Flaserkalk aber, etwa analog dem Namen »Flasergneis «, auf tektonisch, also sekundär gebildete Varietäten « anwenden will, wozú allerdings $z u$ bemerken ist, daß derartige rein durch tektonische Vorgänge entstandene Kramenzelkalke noch nicht bekannt geworden sind. Der. 


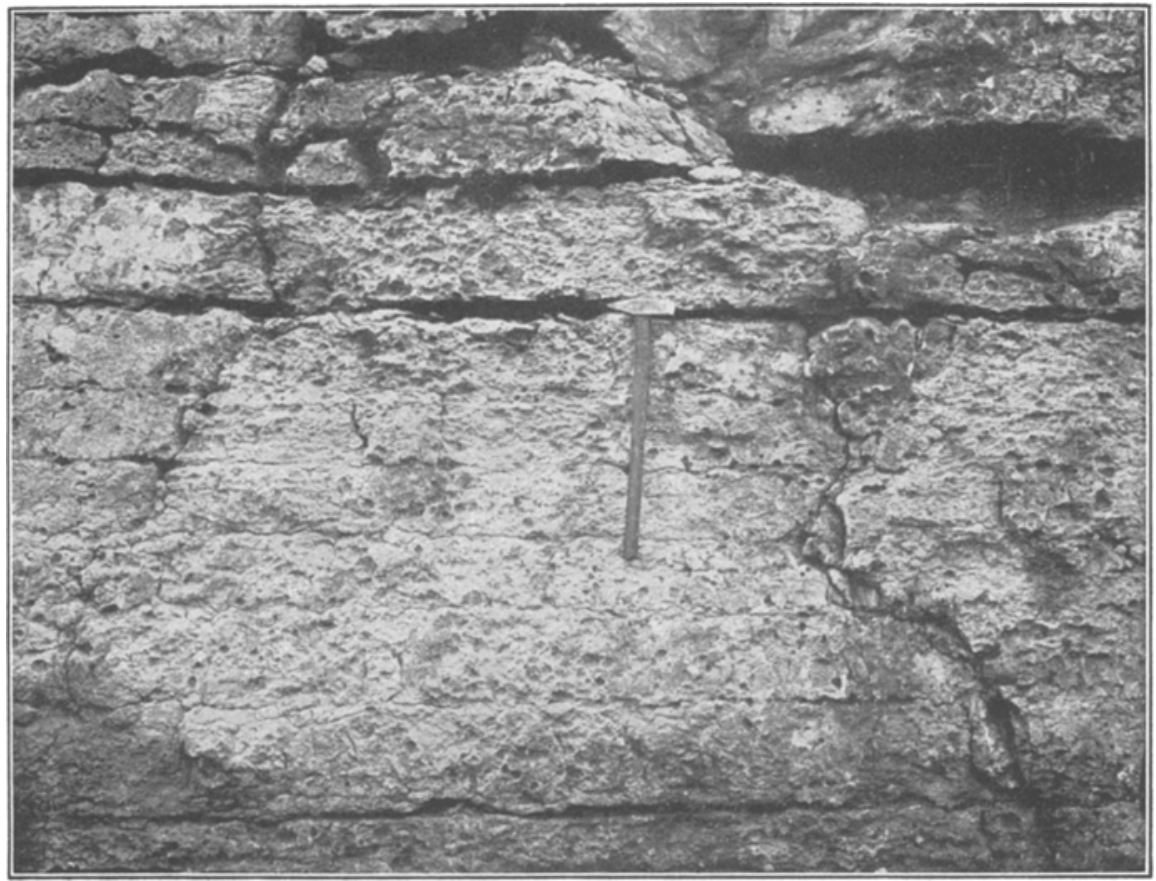

phot. Dr. Schdwf.

Fig. 1.

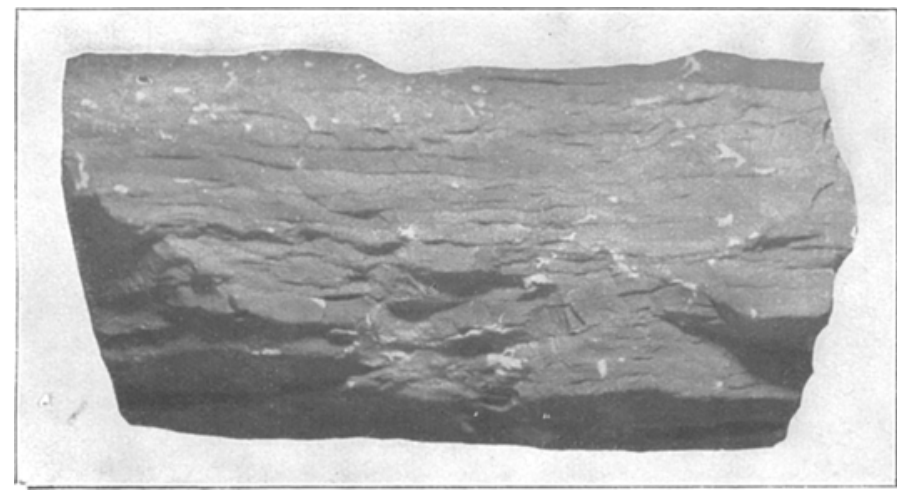

phot. Dr. Schdwf.

Fig. 2. 


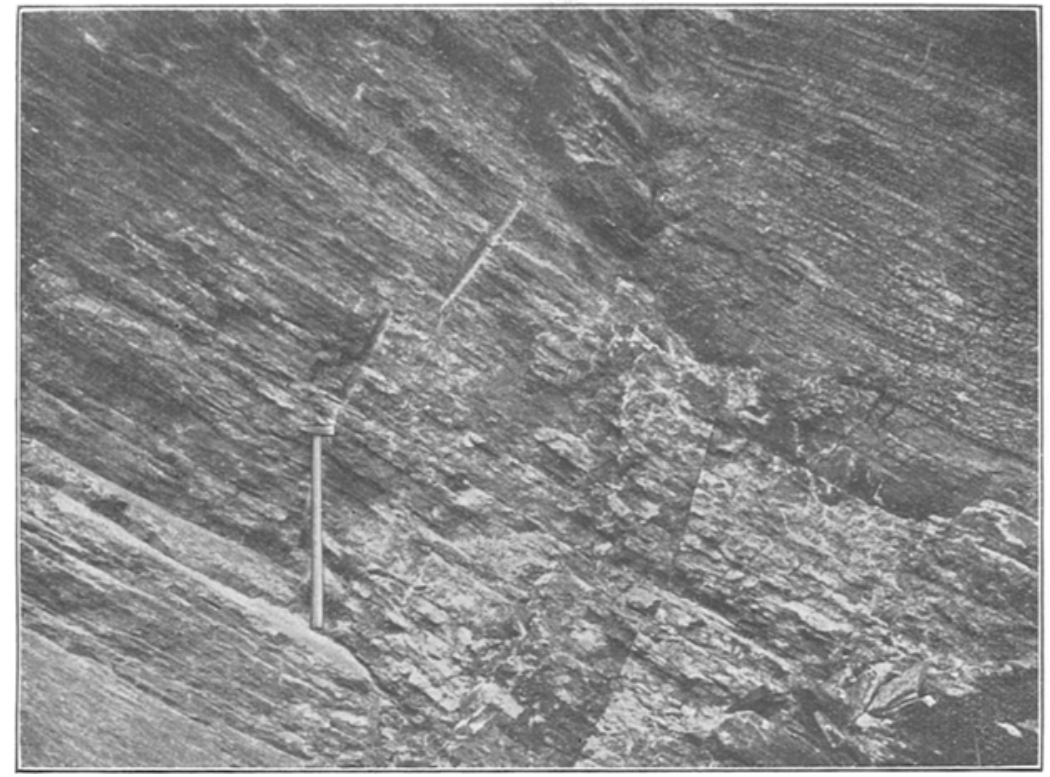

phot. Dr. Sebdwt.

Fig. 1.

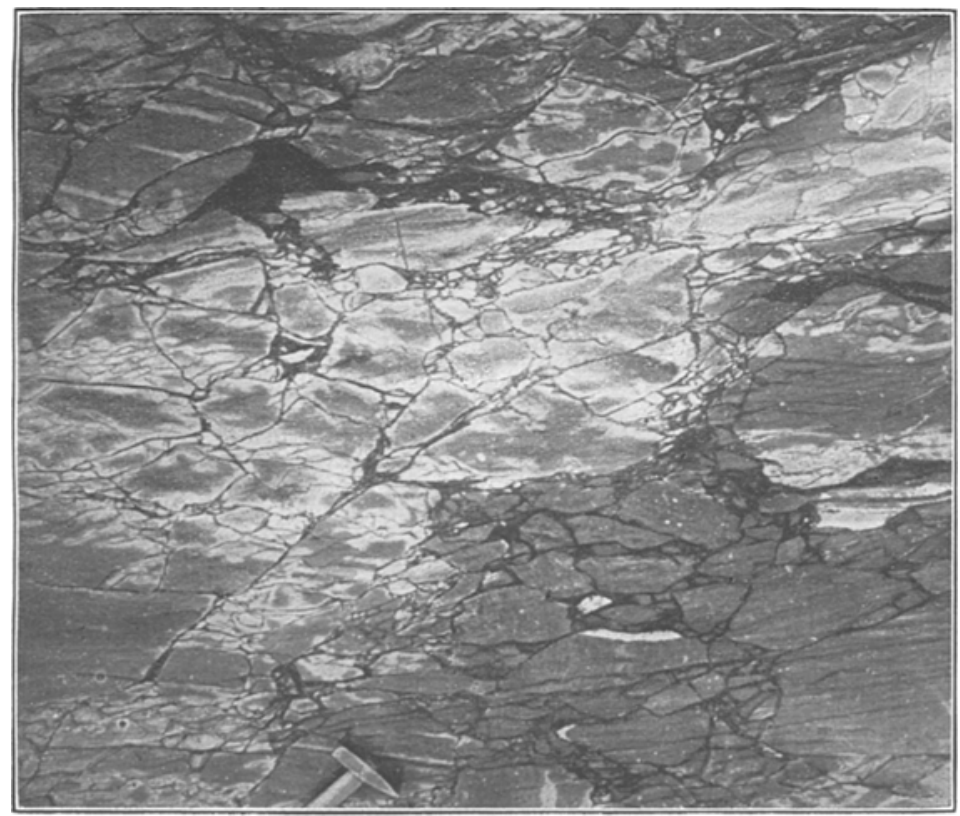

phot, Dr. Schdwf.

Fig. 2. 
Terminus »Kramenzelkalk «, dem keine bestimmte genetische Bedeutung anhaftet, könnte alsdann als Sammelbegriff der einzelnen Typen dienen.

Damit komme ich zu dem dieser Arbeit vorangestellten Motto zurück, das auch in unserem Falle vor einer Verallgemeinerung von Erklärungsweisen für die Erscheinungen warnen möge, die die Natur sauf gar verschiedene Weise hervorzubringen imstande ist «.

Zum SchluB ist es mir eine angenehme Pflicht, den Herren Proff. WEDEKIND- und WEIGEL-Marburg für ihre mannigfachen Ratschläge zu danken, die sie mir bei der Abfassung dieser Schrift in liebenswürdigster Weise zuteil werden ließen.

\section{Erläułerungen zu Tafel I und II.}

Tä. I, Fig. 1. Kramenzelkalk im untersilurischen Echinosphäritenkalk No. von Reval (Esthland). (Vgl, S. 27.)

Fig. 2. Gesteinsstück mit deutlicher vorwiegend parallel angeordneter Flaserung aus der oberdevonischen Kalkbreccie von Horwagen i. Ofr. Ca. 2/3 nat. Gr. In angewittertem Zustande stellen sich derartige Gesteine als typische Flaserkalke dar. (Vgl. S. 28.)

Taf. II, Fig. 1. Oberdevonischer Kramenzelkalk von Görkwitz bei Schleiz i. Th. Im frischen Bruche (linke Hälfte des Bildes) besitzen die Gesteine plattenkalkähnliche Struktur, in angewittertem Zustande (rechts oben) zeigen sie dagegen deutliche Kramenzelstruktur.

Fig. 2. Oberdevonische Kalkbreccie von Horwagen i. Ofr. Die einzelnen Komponenten der Breccie bestehen aus typischem. Flaserkalk. (Vgl. S. 28 und Taf. I, Fig. 2.)

\section{Über nachtertiäre Faltenbewegungen in Albanien.}

\section{Von Ernst Nowak (Leoben).}

(Mit 2 Skizzen.)

Mehrmonatige geologische Kartierungsarbeiten in Albanien während der Kriegszeit ließen mich vor allem den aus den jüngsten Ablagerungen zuisammengesetzten küstennahen Anteil dieses Landes, das sogen. NiederAlbanien, zwischen den Flüssen Ischmi im $\mathrm{N}$ und Vojusa im $\mathrm{S}$ eingehend kennen lernen. Da die Veröffentlichung der Gesamtergebnisse in der heutigen Zeit mit großen Schwierigkeiten zu kämpfen hat und wohl erst in geraumer Zeit wird erfolgen können, möchte ich hier eine Reihe von Beobachtungen aus dem Kapitel Tektonik herausgreifen, die mir von allgemeinerem Interesse zu sein scheinen, um so mehr, als das Problem, das angeschnitten werden soll, in letzter Zeit mehrfach berührt wurde und meine Beobachtungen vielleicht einen kleinen Beitrag zu dessen Klärung liefern können. 